\title{
Pixelated Positron Timing Counter with SiPM-readout Scintillator for MEG II experiment
}

\author{
Miki Nishimura ${ }^{* a}$, Gianluigi Boca ${ }^{b c}$, Paolo Walter Cattaneo ${ }^{b}$, Matteo De Gerone ${ }^{d}$, \\ Flavio Gatti ${ }^{d e}$, Wataru Ootani ${ }^{a}$, Giulio Pizzigoni ${ }^{d}$, Massimo Rossella ${ }^{b}$, Naoya \\ Shibata $^{a}$, Marcello Simonetta $^{b c}$, Yusuke Uchiyama ${ }^{a}$, Kohei Yoshida $^{a}$ \\ The University of Tokyo ${ }^{a}$, INFN Pavia ${ }^{b}$, Dipartimento di Fisicà dell'Università di Pavia ${ }^{c}$, INFN $^{\prime}$ \\ Genova $^{d}$, Università degli Studi di Genova ${ }^{e}$ \\ E-mail: nmiki@icepp.s.u-tokyo.ac.jp
}

\begin{abstract}
In this paper, we introduce the positron timing counter (TC) for the MEG II experiment as an application of Silicon PhotoMultipliers (SiPM) to high-resolution timing measurement. MEG II will search for the $\mu \rightarrow \mathrm{e} \gamma$ decay and needs a precise measurement of the positron timing. The TC is segmented in 512 counters, composed of a scintillator plate readout by SiPMs, to obtain multiple hit positron timing simultaneously such to achieve an excellent overall timing resolution of $\sim 30$ ps. We performed single counter R\&D to optimize the choice of the SiPM manufacturer, the number of the SiPMs, and their connection. To obtain the best resolution, we decided to employ AdvanSiD SiPMs, six of which are attached at both ends connected in series. Moreover we carried out beam tests with 8-9 counters prototypes, where we proved that positron multiple hits improve the resolution according to expectation. The desgin phase of the TC is almost finished and is under construction.
\end{abstract}

International Conference on New Photo-detectors PhotoDet2015,

6-9 July 2015

Moscow, Troitsk, Russia

* Speaker. 


\section{Introduction}

Recently Silicon PhotoMultipliers (SiPMs) have been developed as new photo detectors and are utilized by many experiments. We also employed scintillator counters based on SiPM readout for precise measurement of positron timing in the MEG II experiment.

The MEG II experiment is an upgrade program of the MEG experiment [1] and aims to explore new physics beyond the standard model of particle physics by searching for $\mu^{+} \rightarrow e^{+}+\gamma$ with a world highest sensitivity. The decay is practically forbidden in the Standard Model, while it is predicted by many new physics scenarios beyond the Standard Model. The decay is a simple twobody decay, in which the positron and the $\gamma$-ray are emitted simultaneously back-to-back with the same energy of $52.83 \mathrm{MeV}$, equal to half of the muon mass, in the rest frame of the muon. In contrast the dominant background is an accidental coincidence of a positron from Michel muon decay and an uncorrelated $\gamma$-ray. Therefore a search for $\mu^{+} \rightarrow e^{+} \gamma$ decay requires high precision measurements of time, energy and emission angle for both the positron and the $\gamma$-ray.

Thus positron timing counter (TC) is one of the main detectors for the MEG II experiment. In the MEG experiment we used PMTs for the TC, while in MEG II we will use SiPMs, whose compact size allows us to make smaller counters and finer segmentation. As a result, we can realize positron timing measurement with high precision. In this paper we introduce the detector concept at first and then show several results of counter R\&D including beam test.

\section{New Positron Timing Counter}

The TC is composed of 512 small counters each of which consists of a fast plastic scintillator plate, of two different sizes 120x4(5)0x $5 \mathrm{~mm}^{3}$, with $6 \mathrm{SiPMs}$ at both ends, while in the MEG experiment the TC consists of 15 scintillator bars $\left(800 \times 5 \times 5 \mathrm{~mm}^{3}\right)$ with 2 PMTs (HAMAMATSU R5924). This innovative "pixelated" design of the TC allows simultaneous measurements of positron time to obtain a better overall resolution. The overall resolution can be written as a function of a number of hits as following:

$$
\sigma_{\text {total }}^{2}\left(N_{\text {hit }}\right)=\frac{\sigma_{\text {single }}^{2}}{N_{\text {hit }}}+\frac{\sigma_{\text {inter-pixel }}^{2}}{N_{\text {hit }}}+\sigma_{\text {MS }}^{2}\left(N_{\text {hit }}\right),
$$

where $N_{\text {hit }}$ is number of hits, $\sigma_{\text {single }}$ is the single counter resolution, $\sigma_{\text {inter-pixel }}$ is inter-counter jitter from electronics chain. $\sigma_{\mathrm{MS}}$ is the effect of the multiple scattering. Increasing the number of hits improves the overall resolution as demonstrated in Fig. 1 showing the number of counters hit by positrons in $\mathrm{MC}$ and the overall resolutions as a function of the number of hits. On average, the positrons hit nine counters and the overall timing resolution is expected $\sim 30 \mathrm{ps}$.

\section{Single Counter R\&D}

The first step in optimizing the TC performance is achieving the ultimate timing resolution with single counter [2, 3]. The key is in Fig.2 which is from beam test results discussed in Sec. 4. It shows the energy deposit dependence of the resolution and the energy deposit is proportional to the number of detected photo-electrons. Since the single counter resolution strongly depends on the number of detected photo-electrons, what we should do is to detect as many scntillation photons 

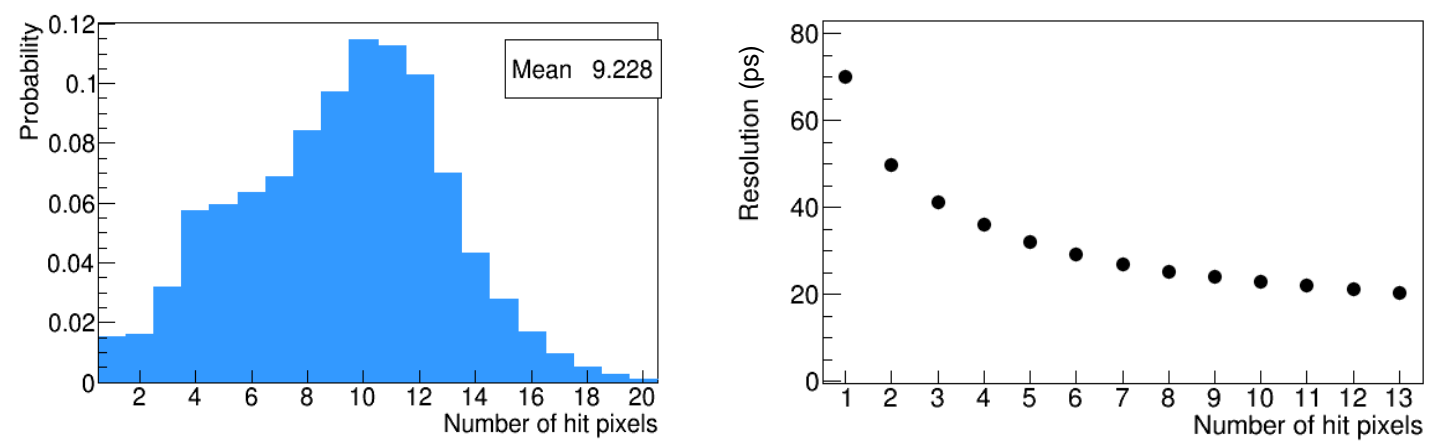

Figure 1: Left: Number of the hit counters (MC). Right: Overall resolution as a function of the number of the hit counters.

as possible. To realize it we carried out several single counter tests with prototypes. In this section, we will show the results focusing on SiPM study.

The simplest idea to collect many scntillation photons is to put SiPMs as much as possible. On the other hand, SiPM cannot be readout individually to avoid increasing the number of output channels. We tested single counters with two options to connect several SiPMs: the series and the parallel connections.

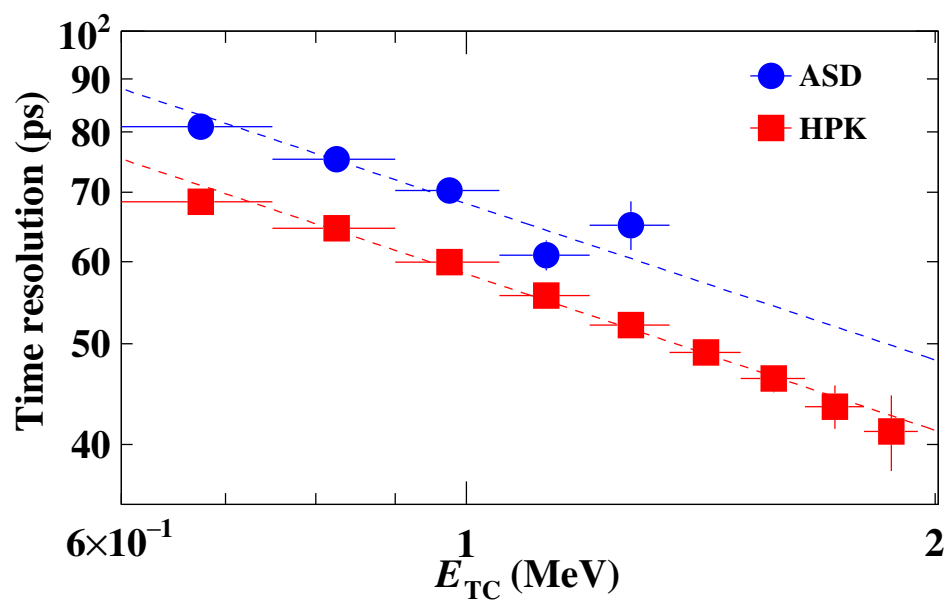

Figure 2: Dependence on the deposit energy of the time resolution. Red markers show HAMAMATSU (S10943-2547(X)) SiPM results. Blue markers show AdvanSiD (NUV type) SiPM results. Scintillator is 90x40x5 $\mathrm{mm}^{3}$ of BC418.

The set up for a single counter test is shown in Fig. 3. We measured electrons from $\beta$-decay of ${ }^{90} \mathrm{Sr}$ with prototype counter. For trigger and collimation a small counter $\left(5 \times 5 \times 5 \mathrm{~cm}^{3} \mathrm{BC} 422\right.$, one HAMAMATSU $3 \times 3 \mathrm{SiPM}$ ) was put behind the prototype counter. All signals are transported to amplifier through 7.4 meter cables and shaped with pole zero cancellation to obtain sharp and short tail signals. Finally signals are digitized by DRS4 [4] at 1.6-5 GHz sampling speed. In Fig. 4, the signals from a counter, which has 3 HAMAMATSU SiPMs (S10362-33-050C) at the each side, are 
shown. Scintillator for the counter is $60 \times 40 \times 5 \mathrm{~cm}^{3}$ of BC422. The waveform of the SiPMs in series connection becomes sharper than that in parallel connection because the overall capacitance in the series is smaller than that in parallel. As a result, the timing resolution with the series connection is better than that with the parallel connection as shown in Fig. 5. Therefore we employed the series connection for the TC.

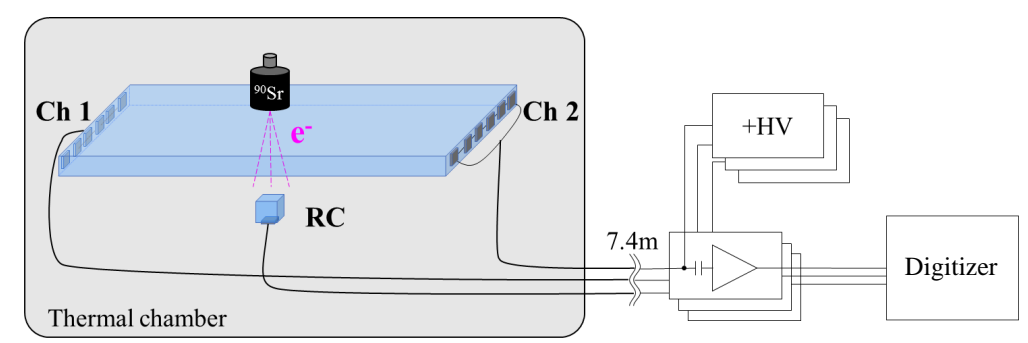

Figure 3: The set up for a single counter test. Counters are inside a thermal chamber. The signals go through $7.4 \mathrm{~m}$ cables and amplifier, then they are digitized.
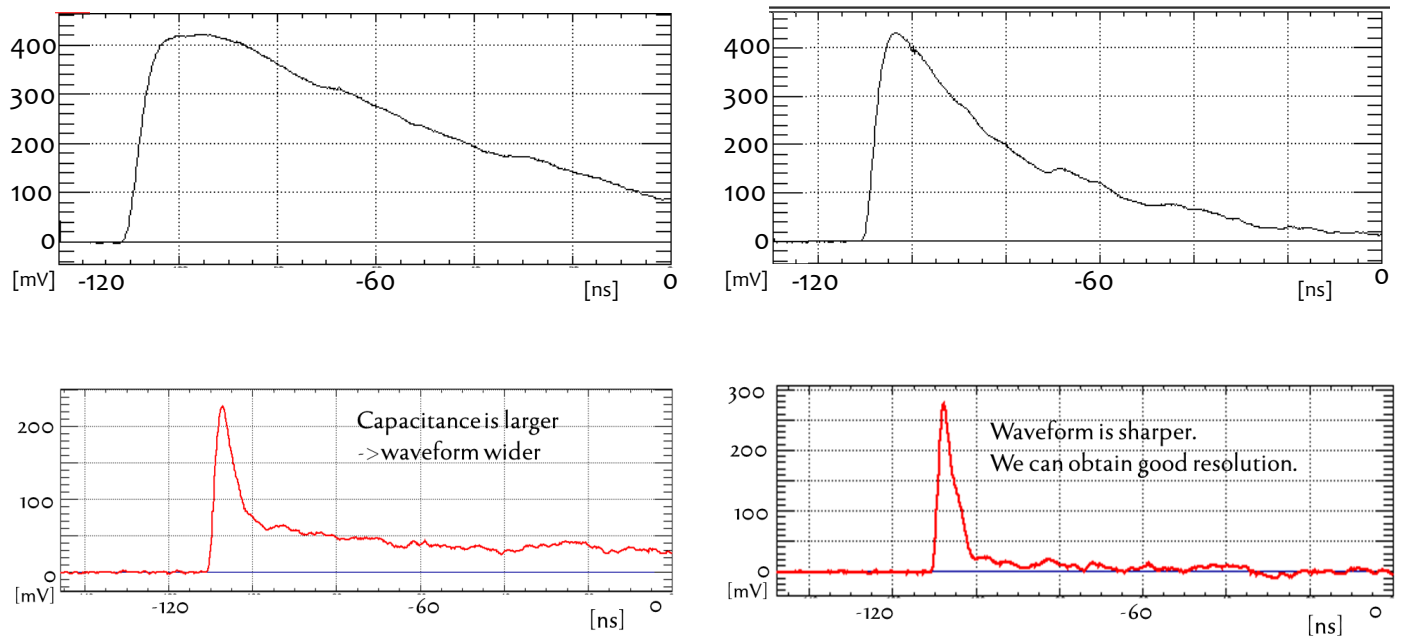

Figure 4: Top: Waveform before shaping. Bottom: Waveform after shaping. Left: Waveform with parallel connection. Right: Waveform with series connection. SiPMs are HAMAMATSU S10362-33-050C. Scintillator is $60 \times 30 \times 5 \mathrm{~mm}^{3}$ of $\mathrm{BC} 422$

We also compared several SiPM's manufacturers. All tested samples are $3 \times 3 \mathrm{~mm}^{2}$. They are HAMAMATSU old type (S10362-33-050C), HAMAMATSU new type (S12572-050C(X), S12572-020C(X)), HAMAMATSU trench type (S12652-050(X)), AdvanSiD (NUV type), KETEK (PM3350 prototype-A), and SenceL (MicroFB-30050-SMT B-Type). In these tests we used BC422 scintillator of $60 \times 30 \times 5 \mathrm{~mm}^{3}$. The resolutions are summarized in Fig. 6. Since the PDEs of HAMAMATSU SiPMs are higher than the others, the resolutions with HAMAMATSU are the best.

However we can improve the resolution by increasing the sensor coverage with more SiPMs attached to a counter even if the single counter resolution is slightly worse. In Fig. 7 the timing 


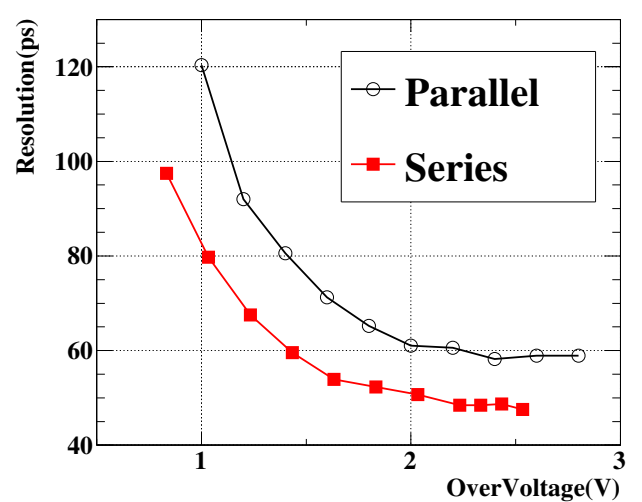

Figure 5: The resolutions of the parallel connection and series connections versus the Over Voltages. SiPMs are HAMAMATSU S10362-33050C. Scintillator is $60 \times 30 \times 5 \mathrm{~mm}^{3}$ of $\mathrm{BC} 422$.

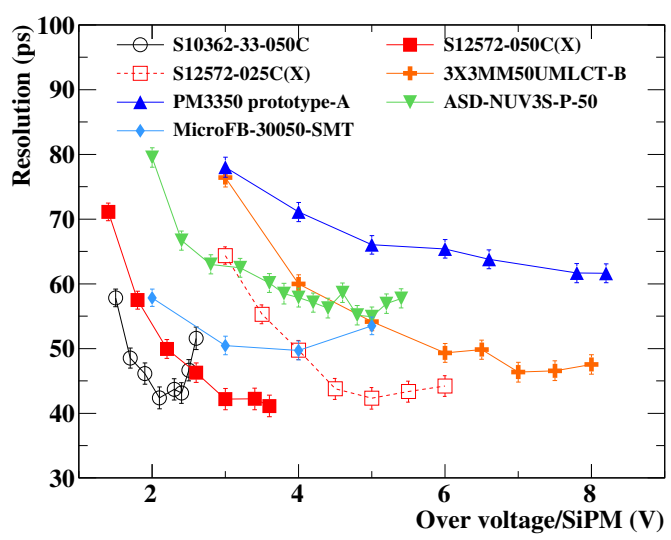

Figure 6: Dependence of the timing resolution versus Over Voltage for different manufacturers. The counter has 3 SiPMs at both ends. Scintillator is $60 \times 30 \times 5 \mathrm{~mm}^{3}$ of BC422.

resolution versus Over Voltage for different numbers of SiPMs with 90x30x5 mm $\mathrm{mm}^{3}$ BC422 scintillator for AdvanSiD SiPMs is shown. The more SiPMs are attached to the scintillator, the better the resolution is. Thus the resolution with 6 AdvanSiD counter (50 ps) is better than that with 3 HAMAMATSU counters (58 ps). From cost point of view, we decided to use AdvanSiD SiPMs and attach 6 SiPMs at both ends of a counter.

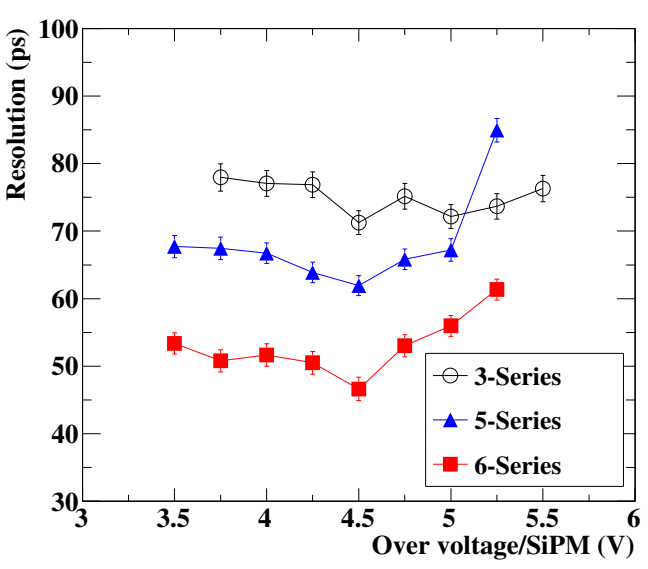

Figure 7: The resolutions with 3, 5, and 6 AdvanSiD SiPMs in series connection. Scintillator is $90 \times 30 \times 5 \mathrm{~mm}^{3}$ of $\mathrm{BC} 422$

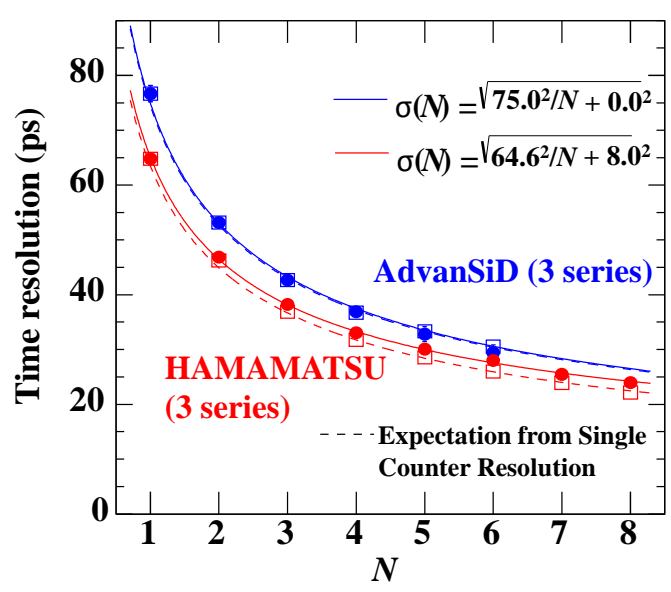

Figure 8: The resolutions as a function of the number of hits in a beam test at BTF. Red markers: HAMAMATSU (S10943-2547(X)). Blue markers: AdvanSiD (NUV type). Scintillator is $90 \times 40 \times 5 \mathrm{~mm}^{3}$ of $\mathrm{BC} 418$

The design of single counter parameters was fixed after several tests to optimize the number of photo-electrons and a better resolution. Finally we decided to employ AdvanSiD SiPMs with

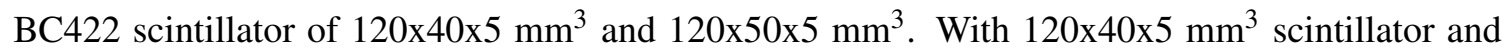


6-series-AdvanSiD we obtained the single counter resolution of $65 \mathrm{ps.}$

\section{Beam Tests}

The main concept of the TC, obtaining an excellent overall resolution by simultaneous timing measurements with multiple counters, was proved by three beam tests. Two of them was carried out at the Beam Test Facility (BTF) of the INFN Laboratori Nazionali di Frascati in Italy [5]. A monochromatic $48 \mathrm{MeV}$ positron beam was used. We prepared 8-9 prototype counters in a background free environment where there are 1-3 positrons in a bunch at $50 \mathrm{~Hz}$. Straight positrons without magnetic field impinge on the prototype counters. We also prepared a reference counter and the timing resolution was calculated by the difference between prototype and reference counters. In Fig. 8 the overall resolution as a function of number of hits is shown. We can see improvement of the timing resolution with $1 / \sqrt{N_{\text {hit }}}$. For example a prototype counter with 3 HAMAMATSU $(\mathrm{S} 10943-2547(\mathrm{X})) \mathrm{SiPMs}$ has the resolution of $65 \mathrm{ps}$. The overall expected resolution with 8 counters is 23 ps. In fact we obtained consistent result, which is 25 ps with 8 counters. In these beam tests, we proved that multiple hits lead to a timing resolution improvement with the number of hit counters.

In the other beam test the rate dependence of the multiple hit scheme was studied. It was carried out in Paul Scherrer Institut. The set up of the prototype counters is similar to previous beam tests. We used the same muon beam line as MEG II but without magnetic field. Prototype counters are hit by a positron generated by muon Michel decay. The positron rate for single counter in MEG II is calculated by Monte Carlo simulation, and is $70 \mathrm{kHz}$ on average. Therefore we adjusted the positron rate to be close to the one expected in MEG II. We tested prototype counters at the expected rate $(53-166 \mathrm{kHz})$, lower rate $(18-64 \mathrm{kHz})$, and higher rate $(89-290 \mathrm{kHz})$. Note that the hit rate depends on the position of the counter. The resolution improvement is visible at all rates in Fig. 9. Small degradation of the resolution compared to the expectation was observed at the higher rate. However at the expected rate it is negligible and the validity of the multiple hit scheme is confirmed at the MEG II environment.

In this beam test we used positrons without magnetic fields. However we plan an engineering run in December 2015 to study the detector performance in the magnetic field and rate of the MEG II experiment. Now we are already in the construction phase of the TC. We are making 512 counters and testing them toward the engineering run.

\section{Summary}

We introduce the concept of the positron timing counter for MEG II in this paper. "Pixelated" design can be realized by small counters with SiPMs. Thanks to this novel design each positron hits several counters, as a result the overall resolution can be improved. To realize TC, we carried out a systematic R\&D. First we optimized the single counter design such as the SiPM Manufacturer, the number of SiPMs and their connection. In addition, beam tests with 8-9 counters confirmed the expected resolution with multiple counter hits even at the rate expected in MEG II. Thus we confirmed the TC basic system and the construction is ongoing toward the engineering run in December 2015 to confirm its performance in the experimental environment. 

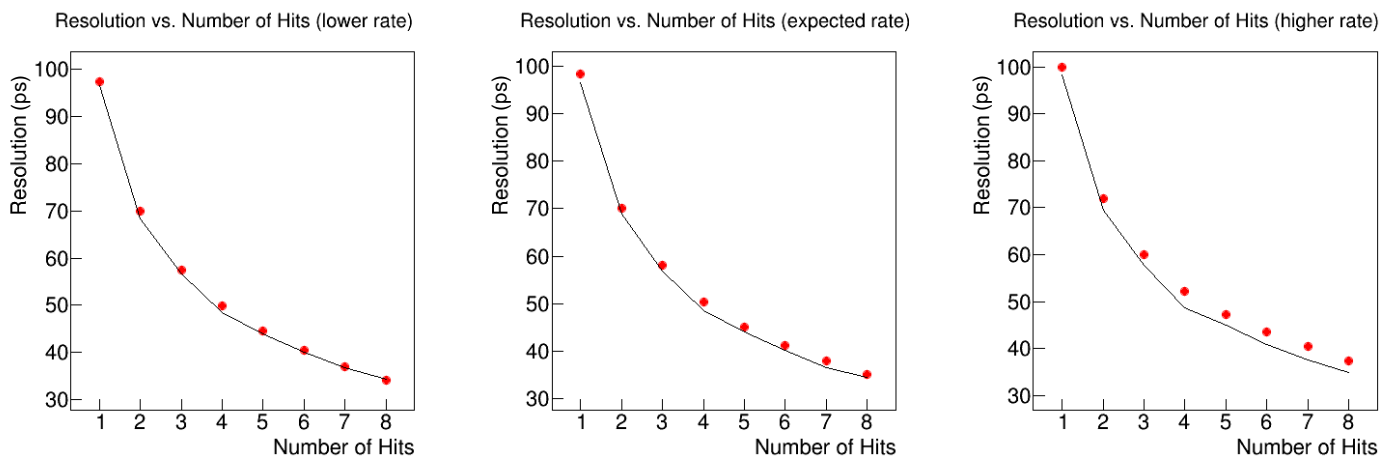

Figure 9: The resolutions as a function of the number of hit counters at lower (18 - $64 \mathrm{kHz})$, expected (53 - $166 \mathrm{kHz})$, and higher $(89-290 \mathrm{kHz})$ rate. Red markers show data. Solid lines show the expectation from single counter resolution. Scintillator is $120 \times 40 \times 5 \mathrm{~mm}^{3}$ and 120x50x5 mm $\mathrm{mm}^{3}$ of EJ232. Each counter has 6 SiPMs (AdvanSiD NUV type) at both ends. Note: Since there was problem in the production of SiPMs in AdvanSiD, the obtained resolution of a single counter is worse then expected.

\section{References}

[1] J. Adam et.al., Phys. Rev. Lett.110.201801 (2013).

[2] M. De Gerone et al., J. Instrum. 9 (2014) C02035. arXiv:1312.0871,

[3] P.W. Cattaneo,M. et al., IEEE Trans. Nucl. Sci. 61 (5) (2014) 2657-2666. arXiv:1402.1404.

[4] S. Ritt et al., NIM-A, 623, pp. 486-488, Nov. (2010).

[5] G Mazzitelli, A Ghigo, F Sannibale, P Valente, G Vignola, Nucl. Instr. and Meth. A, 515(3):524, 2003. 